\begin{tabular}{l} 
journal homepage: http://ijiemjournal. uns.ac.rs/ \\
International Journal of Industrial \\
Engineering and Management \\
volume $13 /$ No $1 /$ March $2022 / 38-48$ \\
\hline
\end{tabular}

Review article

\title{
Industry 4.0 enabling technologies for increasing operational flexibility in final assembly
}

\author{
O. Salunkhe ${ }^{\mathrm{a} *}$ and $\AA$. F. Berglund ${ }^{\mathrm{a}, \mathrm{b}}$ \\ a Department of Industrial and Materials Science, Chalmers University of Technology, Gothenburg, Sweden; \\ b Stena Recycling International AB, Gothenburg, Sweden
}

\begin{abstract}
A B STRACT
The manufacturing industry is facing uncertainties caused by growing competition and increasing customer demands. Simultaneously, the fourth industrial revolution, commonly referred to as Industry 4.0, is helping in modernising the manufacturing industry. In the process of modernising, companies are now capable of building resilience into their systems. This resilience is in the form of higher operational flexibility, which helps cope with the growing uncertainties. The new technologies under the Industry 4.0 umbrella can be used to increase operational flexibility. This article summarises various Industry 4.0 enabling technologies that can increase operational flexibility in final assembly.
\end{abstract}

\section{ARTICLE INFO}

Article history:

Received August 13, 2021

Revised December 27, 2021

Accepted January 8, 2022

Published online January 14, 2022

Keywords:
Operational flexibility;
Final assembly;
Industry 4.0

${ }^{*}$ Corresponding author:
Omkar Salunkhe
omkar.salunkhe@chalmers.se

\section{Introduction}

The Covid-19 pandemic has highlighted the necessity of resilient and highly flexible manufacturing systems. Environmental uncertainties such as the pandemic combined with changing customer demands and high competition push companies to be more flexible with their operating and routing procedures and go from a focused-factory to a reconfigurable factory [1]. Manufacturing flexibility, defined as the ability to cope with changing circumstances or instability caused by the environment, was a scorching topic at the end of the 1980s [2] and the beginning of the 90s. The focus was to define a taxonomy [3]for measuring the effect of different types of flexibilities and how different types of flexibilities affected each other [3]. Then, significance was given to handle manufacturing flexibility, focusing on strategy [4], organisation, and management [4]. The complexity of organisational structures and cultures makes it hard to simultaneously cope with all types of flexibilities. Therefore, this article will only focus on operational flexibility that can be seen as part of the manufacturing flexibility, concentrating on the digital and technological capabilities in a system. A significant share of research on operational flexibility is occupied by topics that closely relate to operational management and managerial decisions. Though these topics remain vital to 
the research, new pathways are now required to examine the implications of the technological aspects on operational flexibility. This article aims to take a step in that direction. This article does not study the economic implications of implementing new technologies and their trade-off in increasing operational flexibility. This lack of economic implications presents an opportunity for further research in this area. The applications presented in this article provide an overview for increasing the operational flexibility in final assembly.

Operational flexibility is most commonly referred to as the system's ability to react to changing environments [5] and can be divided into two contexts; Part/ product and process, defined in Table 1 [6]-[9]. These abilities are based on the technical capability to produce a given set of parts by using alternating machines, alternate processes, and alternate resources [3], [6]. Operational flexibility is often the cumulative effect of different types of flexibility, e.g. routing flexibility, volume flexibility.

At the beginning of the 2010 s, Industry 4.0 was presented as a way to increase manufacturing flexibility through digital capabilities. This digitalisation effort is powered by breakthrough innovations and technologies such as the Industrial Internet of Things (IIoT), Cloud Computing, Artificial Intelligence (AI), Cyber-Physical Systems, Extended Reality (xR), Additive Manufacturing, and Collaborative Robot Applications. These technologies are referred to as the Industry 4.0 enabling technologies supported by new horizontal and vertical integration techniques that help transform traditional systems into Industry 4.0 enabled systems [10], [11]. Considering the cumulative effect of different flexibility types on operational flexibility and the availability of wide-ranging technologies under the Industry 4.0 umbrella, this article aims to answer the following research questions:

1. Which are the best Industry 4.0 enabling technologies for increasing operational flexibility?

2. How can these technologies be used towards increasing the operational flexibility of a final assembly system?
The article's structure is as follows; section two describes the methodology in detail, followed by the description of operational flexibility and its sources in the final assembly in section three. Section four presents different industry 4.0 enabling technologies in detail, followed by a discussion in section five. Finally, the article is concluded in section six.

\section{Methodology}

A literature review is an excellent tool to manage the diversity of knowledge for a topic like flexibility, especially operational flexibility. The literature review in this article follows a systematic review approach. Systematic literature review, as defined by Grant and Booth [12] seeks to systematically draw together all known knowledge on a topic area by adhering to the guidelines on the conduct of a review and aims to answer a particular research question, test hypothesis, and build theories [13]. Tranfield et al. [14] highlight that a systematic review is one of the best methods to produce reliable knowledge in context-sensitive research. Such an approach is necessary for a diverse topic like flexibility with a wide range of available literature. Designing a literature review by narrowing down on a topic using specific guidelines offers a better opportunity to focus on a topic like operation flexibility.

The systematic literature review follows a stepby-step procedure [13]. The systematic literature review presented in this article follows the principles of deductive reasoning. Deductive reasoning uses a hypothesis to confirm or reject a theory [14]. Furthermore, systematic literature reviews aim to answer a particular research question.

The keyword string and the systematic review process is presented in figure 1. In addition, the general methods of content analysis have been followed to review the literature. Articles that included Industry 4.0 and flexibility as buzzwords have not been included in this review. Scopus and Web of Science online databases have been used for retrieving and

Table 1. Definition of Operational Flexibility

Context Description

Part/Product

Ability of the system to produce a part in different ways, such as by using alternative processes and interchanging machines.

Process

Ability of the system to use alternate machines to produce a given part along with the capability of the system to deliver material to produce the given set of parts. 


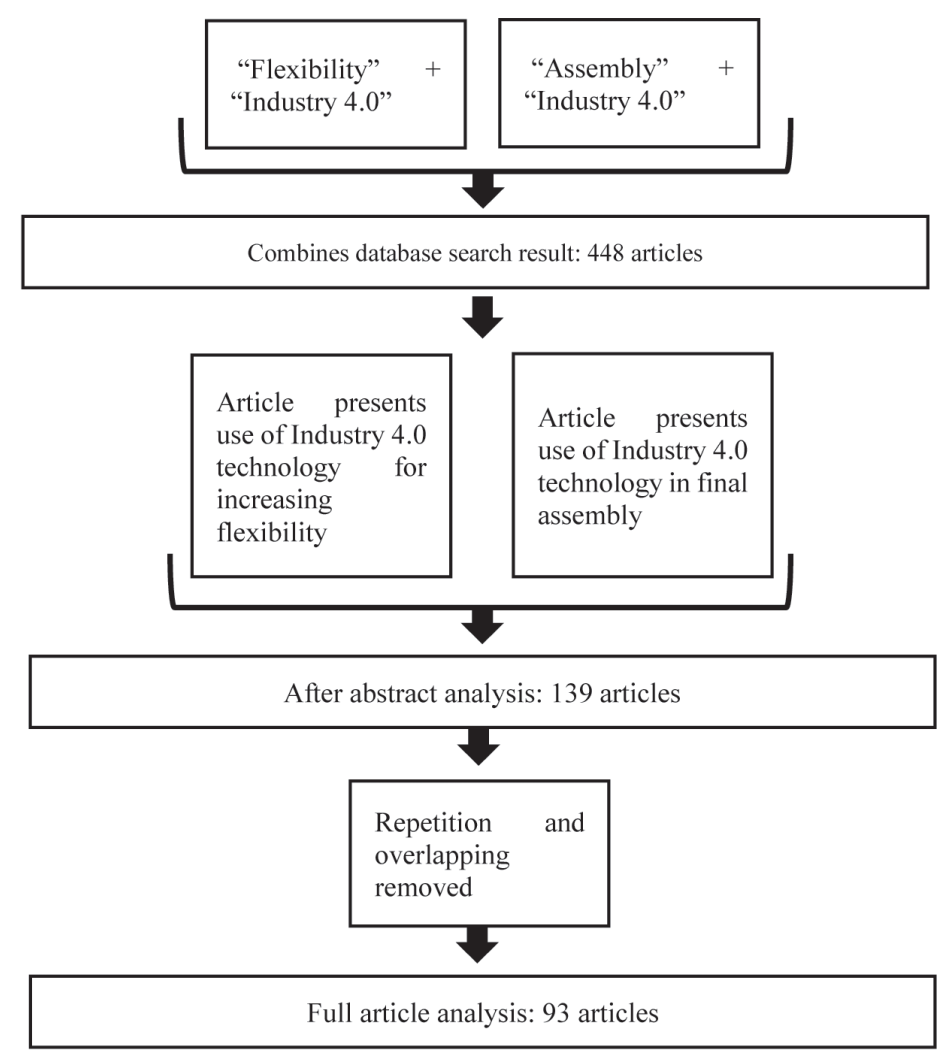

Figure 1. Overview of systematic literature review process

analysing literature. The original search resulted in 448 articles; this number was reduced to 139 after abstract analysis.

After considering the repetition and overlapping of ideas, 93 articles were selected for final analysis. The industry 4.0 enabling technologies identified in the review are shown in figure 2 . The individual details are explained in section 4 .

\section{Operational Flexibility}

The result from the literature shows that several factors contribute to operational flexibility. While Manufacturing flexibility takes a broad scope of the term flexibility, other types of flexibility are a little more narrow. In the 1970s, the concept of flexibility was influenced mainly by the development of $\mathrm{CNC}$ machines that aided the conception of Flexible Manufacturing Systems (FMS), enabling the ability to produce various products on the same manufacturing system [15]. Different flexibility concepts, such as machine flexibility, volume flexibility, product flexibility, and production flexibility, were put forward in the upcoming decade. In the 1990s, increasing globalisation, unpredictable market requirements, and increasingly changing product demands prompted the requirement to improve the design and architec- ture of manufacturing systems to safely respond to uncertainties, thus leading to the development of Reconfigurable Manufacturing Systems (RMS). While FMS enabled by CNC machines leads to versatility in producing different products, RMS facilitated rapid response to market demands and uncertainties. This response was based on RMS's characteristics and principles, such as scalability, convertibility, diagnosability, customisation, modularity, and integrability [16]. El Maraghy [16] also highlighted the continuum of flexible and reconfigurable manufacturing systems. Though FMS provided considerable abilities in achieving flexibility, RMS enhances these capabilities by adding modularity. The modular platforms enabled by RMS were based on reconfigurable machines, the generation of alternative process plans, changeable infrastructure, changeable in-house routing, and intelligent automation based on sensor feedback, adaptive control, and self-reconfiguration potential [5]. Though the technology was not fully developed to achieve these advantages before, the recent developments enabled by industry 4.0 technologies are fully capable of attaining FMS and RMS. The core characteristic of both FMS and RMS is based on flexibility, especially the flexibility of manufacturing operations [5], [16]. In terms of a specific type of flexibility, this flexibility is considered operational flexibility. Different sources of op- 
erational flexibility, as reviewed by $\mathrm{Yu}$ et al. [5] and Jain et al. [17] are shown in figure 2. Their description is provided in Table 2.

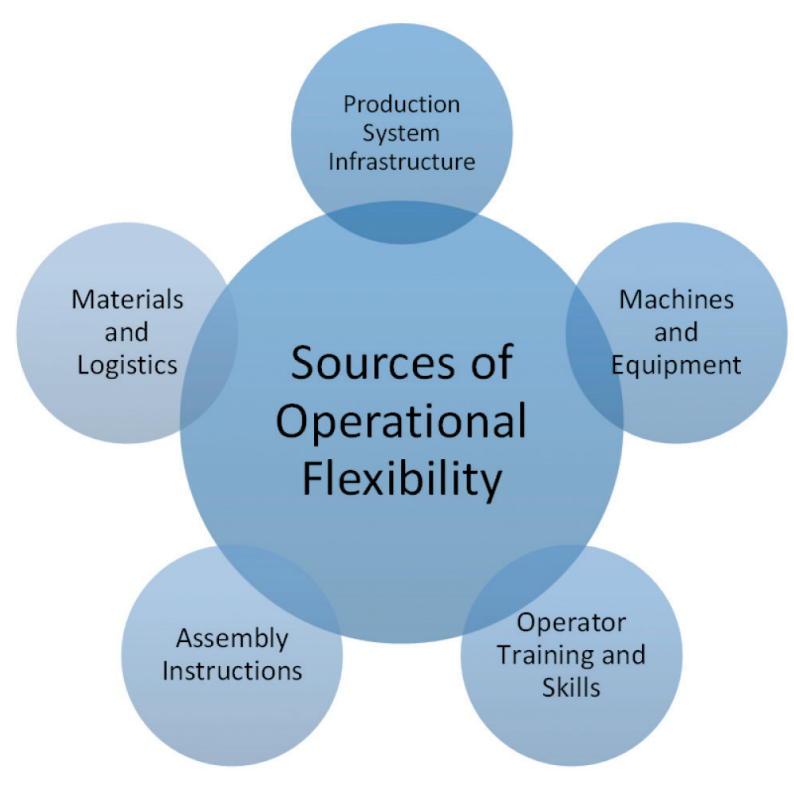

Figure 2. Sources of Operational Flexibility

\section{Industry 4.0 enabling technologies for final assembly}

The enabling technologies of industry 4.0 could all increase the manufacturing flexibility, but a new question occurs compared to FMS and RMS; Can companies implement these enabling technologies everywhere and at the same time? And if not, where should they start? At the beginning of the industry
4.0 era, the focus was on interoperability between different machine systems to enable the FMS and RMS visions. In the last five years, the trend is more towards the early thoughts of manufacturing flexibility in terms of organisation, strategies and new thoughts about resilience, servitisation and humancentric manufacturing [10], [18].

Final Assembly is a process of product realisation in which components, parts in the form of products, or sub-assemblies are integrated to form a final product [19], [20]. The integration process consists of various operations, such as welding, joining and manual assembly. A standard structure of an assembly plant is as follows: stamping, body shop, paint and final assembly [21]. This paper will focus on the final assembly, which is the final stage of the product realisation process with a high added value within the products and with historically low technical and digital capabilities [22], [23]. This area has not been the main focus of industry4.0 realisation. Industry 4.0 enabled systems commonly consist of different enabling technologies integrated to increase the respective systems' efficiency and productivity. The technological integration is vital for a properly functioning industry 4.0 enabled system as the different technologies used in a system need to support each other for data and information transmission. Any operation with high human involvement requires a good balance of technologies that can help increase the operational flexibility of final assembly without causing security and safety risks to the operators. Various such technologies identified in the literature review that can be used to increase the operational flexibility of final assembly are presented in figure 3.

Table 2. Description of Operational Flexibility Sources

\section{Source of operational flexibility Description}

Production system infrastructure

Machines and equipment

Operator training and skills

Assembly instructions

Logistics and material handling
Ease in changing/modifying the layout of the system, ease in integrating new machines and technologies with an existing system.

Different types of machines available, the capability of machines to produce different products, setup time and change over time for machines, availability and reusability of other equipment such as fixtures.

The ability of operators to assemble a wide range of products without defects, operator skills and capability to quickly change stations and their ability to use new technologies and techniques.

Different types of assembly instructions and their method of delivery

The ability of the system to deliver material to workstations in the shortest time and safest possible way 


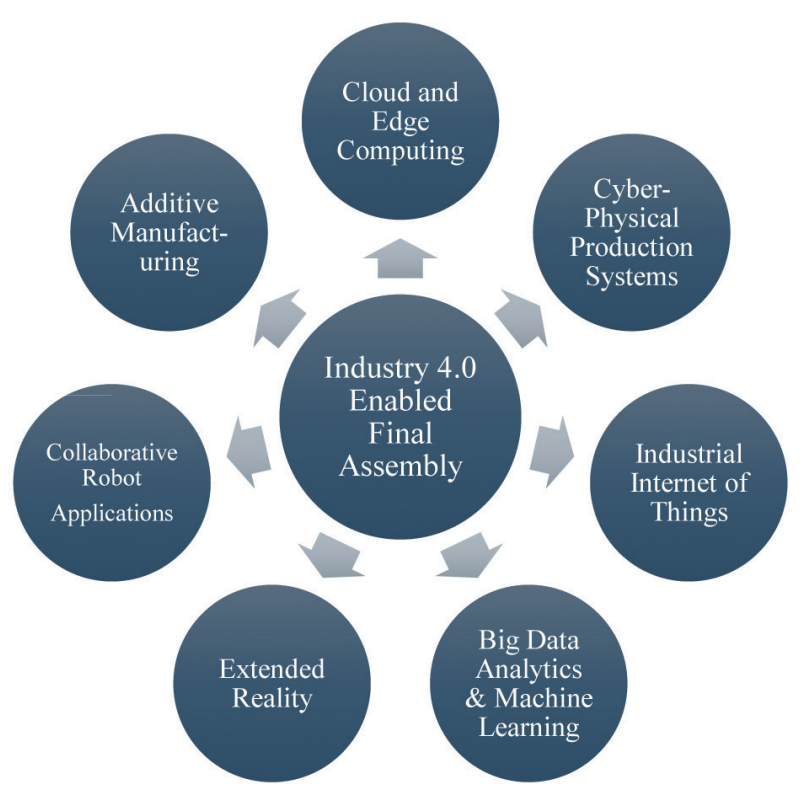

Figure 3. Industry 4.0 Enabling Technologies for Final Assembly

These technologies and their constituting elements, along with their implication on final assembly, are presented below.

\subsection{Additive Manufacturing}

Additive manufacturing is a process of building complex three-dimensional parts in a layer-by-layer manner from their respective CAD models [24]. Material is usually deposited using a printer head above each layer to form a cohesive bond. This layer-by-layer printing process provides the opportunity to print parts that are too complex for traditional manufacturing processes. The printing process also allows the possibility to reinforce a part with different materials to achieve strength and durability. There has been considerable growth in additive manufacturing for producing complex parts. Additive manufacturing is used for the rapid prototyping of products [24] . It is also used for producing customised products [25]. In the final assembly, additive manufacturing can manufacture customised tools and equipment and fixtures.

\subsection{Cloud and Edge Computing}

The National Institute of Standards and Technology (NIST) [26] defines cloud computing as "A model for enabling ubiquitous, convenient, on-demand network access to a shared pool of configurable computing resources (e.g., networks, servers, storage, applications, and services) that can be rapidly provisioned and released with minimal management effort or service provider interaction." Cloud comput- ing aims to provide computing services on-demand with high reliability, scalability, and availability in a distributed environment such as manufacturing [27]. Cloud computing proposes moving from productionoriented manufacturing to service-oriented manufacturing, meaning every resource client can request a service from cloud-based systems. This may include CAD designs, instructions for operators, material requests. [27]. An advantage of this process is storing data such as CAD files and operator instructions at a central data storage and distributing it to different sites quickly and securely. In case of any modification or change in product design, these changes can be rectified in just one place rather than at every site. Data speed and transmission rates are vital for Industry 4.0 enabled systems, Especially in the decision making of autonomous and semiautonomous systems. In edge computing, the data is processed as close to the source as possible using cloud-based resources [28], [29]. This reduces the data transmission time to micro-seconds helping various control systems in a quick decision-making process. For final assembly systems, cloud and edge computing can be instrumental in enabling plug-n-play type CPS-based modularised workstations[30], [31].

\subsection{Cyber-Physical Production Systems}

Cyber-Physical Systems, commonly known as CPS, are autonomous physical systems interconnected with other systems in their surroundings by integrating physical components with the cyber world of networks and computation. These systems can collect, process, and communicate data and information to perform their operations efficiently and intelligently [32]. CPS in manufacturing systems is widely recognised as Cyber-Physical Production Systems (CPPS) [33]. In final systems, individual workstations can function as CPPS modules and enable modularised final systems. These CPPS enabled modules can be added and removed from assembly lines whenever required. Product and process change is more manageable with such modularised assembly. With the capability of self-awareness [34], CPPS modules can reduce system integration time and assist operations within assembly processes [35] by guiding an operator in assembling a product or providing instructions to a collaborative robot.

\subsection{Industrial Internet of things (IIOT)}

As defined by Badarinath et al. [36], the industrial internet of things is an ecosystem of uniquely identifi- 
able physical devices and software components that are interconnected and can transfer data with other devices over a network with limited human intervention. IIoT offers the possibility to remotely monitor and control physical objects such as machines, robots, and processes over a network. IIoT can monitor and optimise assembly processes in final assembly systems by utilising the interconnected devices and systems in its ecosystem. With the wide range of supported protocols, IIoT can connect to legacy devices and the devices running on new protocols making it an ideal middleware for connecting different systems. For example, IIoT supports traditional industrial communication protocols such as $\mathrm{TCP} /$ IP, Modbus, and modern protocols such as UPC UA, NODE-Red. Thus, having good IIoT platforms increases the number of different technologies that can be integrated. IIoT can also manage on-demand material delivery using advanced AGV's [37]. The most advantageous use of IIoT in final assembly is to deliver assembly instructions to operators. IIoT can deliver digital instructions in various formats such as $3 \mathrm{D}$, audio, visual and image-based instructions to operators throughout the assembly systems.

\subsection{Big Data and Machine Learning}

The advanced sensors, actuators, and processes produce a large amount of industrial data. Processing this data offers to build an integrated environment such as a smart factory and provides transparency of production operations [38]. Big data also helps manage and control production processes efficiently based on real-time data [39]. Furthermore, machine learning allows detecting abnormalities and patterns from production data and helps create digital twins [39], [40]. The data generated by the system can be further used to optimise production processes and ensure the high quality of products [39], [41].

\subsection{Extended Reality (XR)}

There are different types of xR technologies, Virtual Reality (VR), Mixed Reality (MR), and Augmented Reality (AR). Extended reality is a general term used to refer to all real and virtual combined environments and human-machine interactions generated by wearables and computer technology [42]. Extended reality is central in enabling industry 4.0 in the final assembly. AR is an interactive experience that combines real and virtual worlds, provides real-time interaction and accurate 3D registration of virtual and real objects [43]. Virtual reality can train new operators and help them acclimatise to the real environment [44]. This can be done by using 3D point cloud data to imitate final assemblies in a $3 \mathrm{D}$ environment and provide operators with the possibility to experience the actual working conditions. Classified by the European Union as an essential technology that will push the development of smart factories, AR can support human operators with an intelligent manufacturing environment. AR also offers a fast and secure method to facilitate interaction and collaboration between production system data and human operators. The use of AR has been increasing in industry and academia; For example, it has been used to provide assembly instructions and train operators [45].

\subsection{Collaborative Robot Applications}

Collaborative robots, commonly known as "Cobots," are a type of industrial robots designed to directly interact with humans in completing a task [46]. They are equipped with advanced sensors and actuators capable of detecting obstructions in their paths. The traditional industrial robots face huge limitations such as a caged area for safety, less flexibility when moving between workstations, extended programming and verification process for their application in the final assembly, and the high involvement of human operators in assembly processes. With their safety features, fast and comparatively easy programming and verifications process, and their ability to work in close proximity with a human operator, Cobots help overcome the challenges industrial robots face [47]. Besides taking over tedious and unergonomic tasks such as pick and place operations, cobots can also be used for material handling in final assembly [48] and quality assurance and verification processes [49].

\section{Discussion}

Various sources and technological capabilities must be facilitated to achieve total operational flexibility. Strategies for implementing technologies are vital in the same way as in the 1970s and the 1990s when implementing manufacturing flexibility in the FMS and RMS. Proof of Concepts (PoC) and Proof of Values ( $\mathrm{PoV})$ will be essential to scale the solution quickly and effectively. However, there is also a need for long-term effects on the implementation of Industry 4.0. Several articles in the literature review showed that implementation looks easy in theory but could be tricky in practice. Such as the use of digital instructions for machine tool setup [45], au- 
tomated dynamic planning and scheduling of production process [31], [50], algorithms for automated line balancing [19], [51], but few articles show fully implementation of operational flexibility with Industry 4.0 digital tools and capabilities. It is important to consider the entire system when implementing an industry 4.0 enabling technology towards increasing operational flexibility [52]. In chapters 3 and 4, the operational flexibility and technical capabilities of industry 4.0 have been discussed. The vital question is how to combine them to increase operational flexibility in final assembly. The sections below will our research questions with the help of two different case scenarios;

\subsection{Case 1: Combining IloT and collaborative robot application}

Starting with the system's infrastructure, using the IIoT platform as the backbone of the assembly system allows seamless data exchange throughout the system. This, combined with CPPS enabled plugand-play workstations, as explained in section 4.3, results in an autonomous and intelligent system. Such a system will help reduce the time required for changing and modifying assembly layout as the CPPS workstations are capable of self-identification and integration. The reduction in time required to make physical changes makes it easier to introduce new products, modify existing products, and upscale and downscale production rates as per the market demands. Adopting such a system helps increase operational flexibility in the context of production system infrastructure.
The IIoT platform can also provide different types of digital instructions to operators. They aid in increasing operational flexibility in the context of assembly instructions. At the same time, CPPS-enabled workstations can be used to integrate new machines and equipment such as collaborative robots into the system without making significant changes in the physical or digital infrastructure. These connections and implications are illustrated in figure 4. Collaborative robots are known for their lightweight and easy installation. This helps move Cobots quickly between different workstations whenever required without making significant changes to workstations or assembly processes.

Most importantly, collaborative robots can take over unergonomic tasks from operators, leading to reduced unergonomic strain on the operators. The cobot takeover also frees the operators to carry out more meaningful tasks. This helps increase the final assembly's operational flexibility and transforms traditional final assembly systems into resilient and human-centric systems capable of sustaining unforeseen circumstances.

\subsection{Case 2: Combining IloT and XR technologies}

Even in a highly automated final assembly, a human operator remains irreplaceable. Enhancing the operators will lead to the enhancement of the final assembly system. Operator capabilities can be developed for increasing operational flexibility by teaching them new skills and techniques and providing them with new tools and equipment. A well-trained and

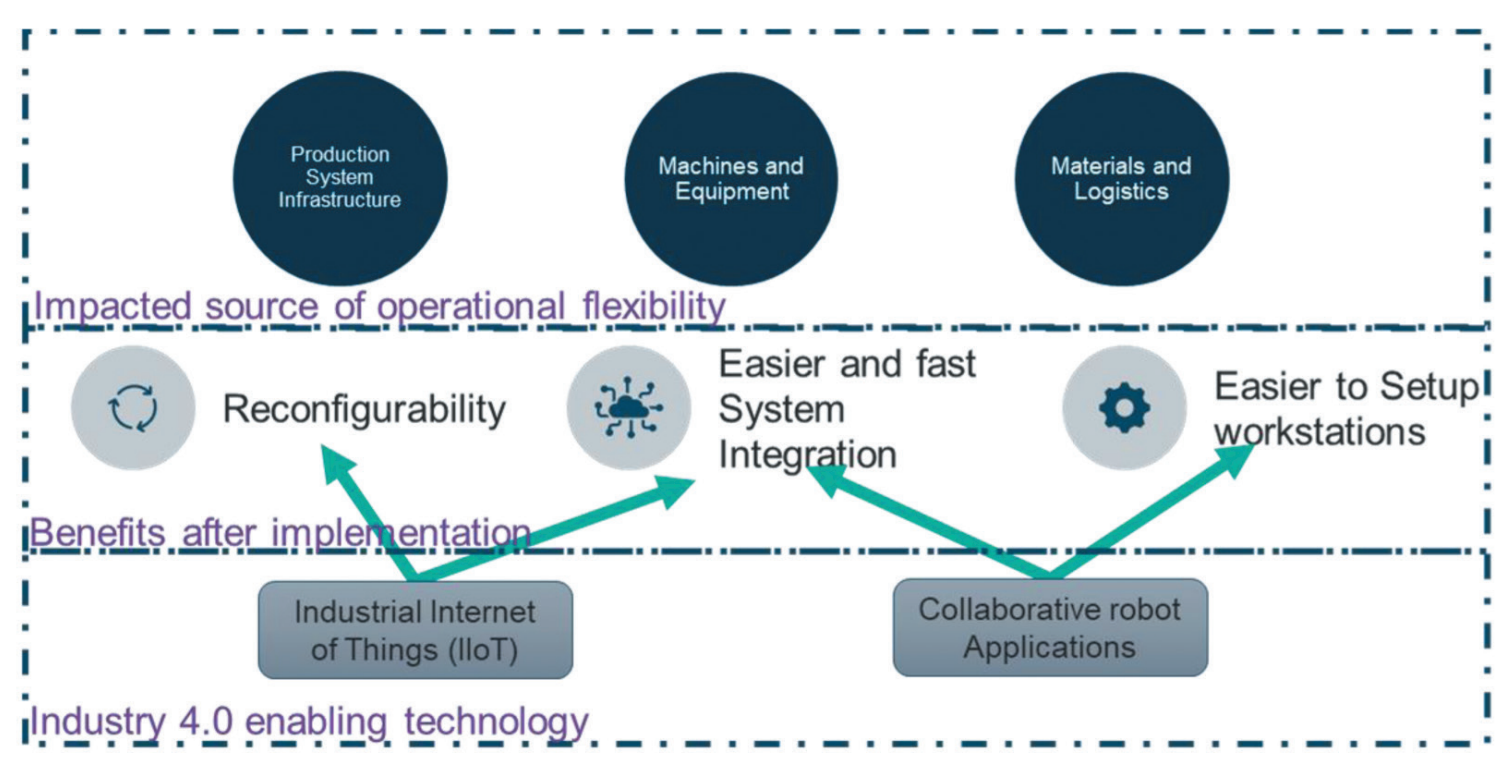

Figure 4. Technology with system-wide implementation and impact on sources of operational flexibility 


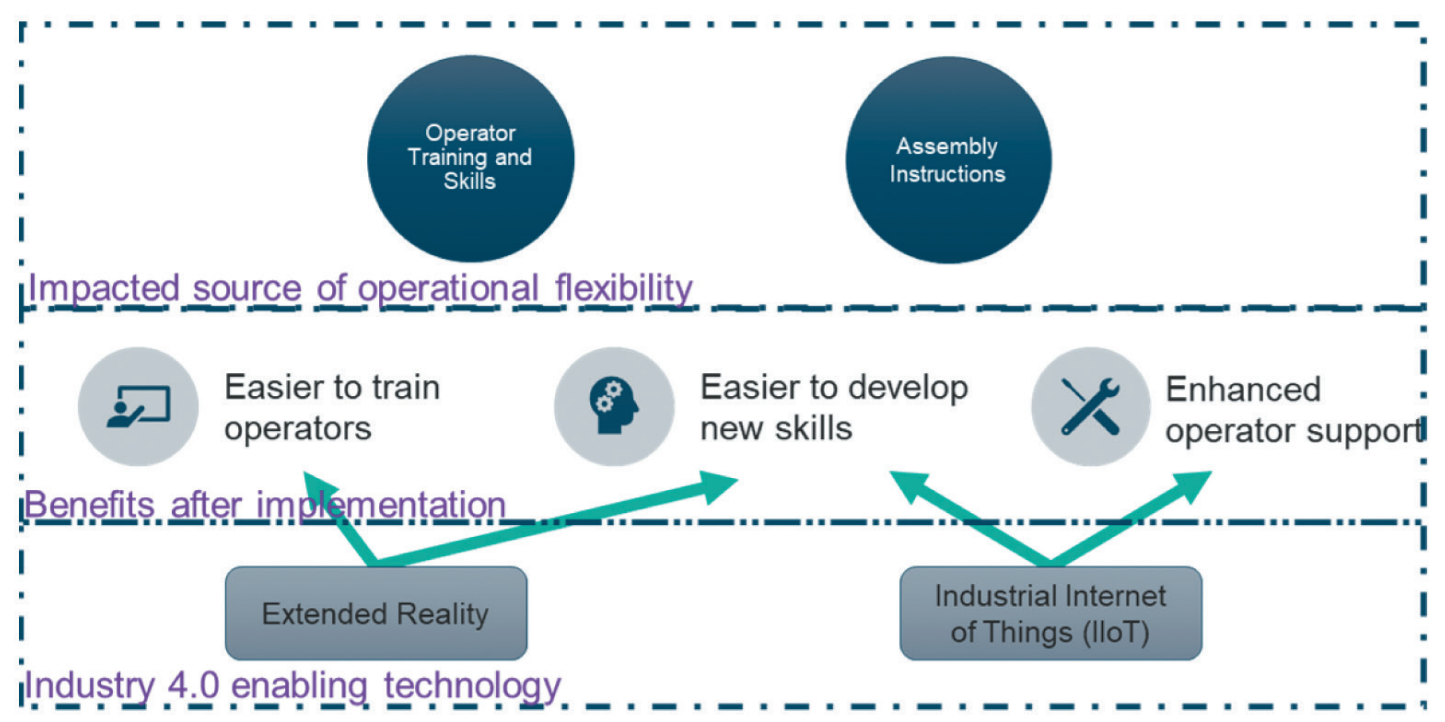

Figure 5. Human-centred technology and impact on sources of operational flexibility

highly skilled operator can quickly adapt to changes in assembly procedures. This helps increase operational flexibility in the context of operator training and skills. This quick adaptation by operators is affected by the instructions the operators receive. Having digital instructions such as audio-visual guides and AR glasses will increase the cognitive ability of operators in understanding different assembly instructions compared to paper-based instructions. Digital instructions will aid the quick adaptation process and increase the operational flexibility of final assembly in the context of assembly instructions and production system infrastructure as operators can now be easily moved in between workstations. An operator can only perform well if he/she is provided with proper training. xR technologies can be used for operator training and skill development.

A highly skilled operator supported by appropriate tools can assemble complex parts quickly and efficiently. Such an approach enables producing customised products with the help of AR instructions. This is illustrated in figure 5. AGV's are capable of handling on-demand material delivery safely and efficiently. Apart from instructions, operators need correct parts for assembly. With a robot mounted AGV, this process can be further automated and optimised. Use of the industry 4.0 enabling technologies such as IIoT platforms, CPPS enabled workstations, collaborative robots and supported by highly skilled operators and an efficient material handling system provides the final assembly with the necessary capability for using alternate processes as well as alternate resources to produce a given set of products, thus increasing the operational flexibility of the final assembly system.

\subsection{Limitations and further research}

Implementing new technologies results in new challenges; some are common while others are unforeseen. There might also be compatibility issues between new and legacy machines and industrial protocols. Such details are not considered in this article. Another limitation can be the hesitation of operators in adapting to new technologies and techniques, which needs further investigation. A challenge for using such modern technologies is that of cybersecurity. Companies must have robust cyber-security teams to secure their networks and provide constant safety from cyber-attacks. Cybersecurity though a vital part of the digital infrastructure, is not in the scope of this article.

\section{Conclusion}

This article presents the use of Industry 4.0 enabling technologies for increasing operational flexibility in final assembly systems. Different sources of operational flexibility are matched with a corresponding industry 4.0 enabling technology. A detailed description of these enabling technologies and their impact on operational flexibility is explained in this article. The impact and dependence of different industry 4.0 enabling technologies on each other and the interconnection between different sources of operational flexibility is clarified in detail. Finally, the need for a holistic approach is highlighted. The implications of this article on scientific and industrial communities are as follows. Based on our findings, combining 
IIoT platform and operators centred technologies such as digitalised assembly instructions, collaborative robot applications, and $\mathrm{xR}$ technologies offer maximum benefits towards increasing operational flexibility in final assembly. The technologies mentioned above help enhance the operators' physical and cognitive capabilities, the most flexible resource in final assembly. This article promotes a new dimension to study the impact of multiple industries 4.0 enabling technologies on each other. By focusing on the final assembly, the authors aim to draw the scientific community's attention to human aspects in operational flexibility and the technological implications on human operators. For the industrial stakeholders, this article provides information on different types of operational flexibility, their inter-linkage, and the different industry 4.0 enabling technologies that can help increase operational flexibility.

\section{Acknowledgements}

This paper is an extended version of the conference paper presented at the 2020 IEEE International Conference on Engineering, Technology, and Innovation (ICE/ITMC) [53].

\section{Funding}

This work was supported by VINNOVA, the Swedish energy agency under the project "Smart Production” [Project ID: 2017-05208].

\section{References}

[1] O. Okorie, R. Subramoniam, F. Charnley, J. Patsavellas, D. Widdifield, and K. Salonitis, "Manufacturing in the Time of COVID-19: An Assessment of Barriers and Enablers," IEEE Engineering Management Review, vol. 48, no. 3, pp. 167-175, Jul. 2020, doi: 10.1109/EMR.2020.3012112.

[2] N. Slack, "The Flexibility of Manufacturing Systems," International Journal of Operations and Production Management, vol. 7, no. 4, pp. 35-45, Apr. 1987, doi: 10.1108/EB054798.

[3] A. K. Sethi and S. P. Sethi, "Flexibility in manufacturing: A survey," International Journal of Flexible Manufacturing Systems, vol. 2, no. 4, pp. 289-328, 1990, doi: 10.1007/ BF00186471.

[4] D. M. Upton, "The Management of Manufacturing Flexibility," California Management Review, vol. 36, no. 2, pp. 72-89, Jan. 1994, doi: 10.2307/41165745.

[5] K. Yu, J. Cadeaux, and B. N. Luo, "Operational flexibility: Review and meta-analysis," International Journal of Production Economics, vol. 169, pp. 190-202, Nov. 2015, doi: 10.1016/J.IJPE.2015.07.035.
[6] M. Pérez-Pérez, A. M. Serrano-Bedia, M. C. LópezFernández, and G. García-Piqueres, "Research opportunities on manufacturing flexibility domain: A review and theory-based research agenda," Journal of Manufacturing Systems, vol. 48, no. May, pp. 9-20, 2018, doi: 10.1016/j.jmsy.2018.05.009.

[7] L. L. Koste and M. K. Malhotra, "Theoretical framework for analysing the dimensions of manufacturing flexibility," Journal of Operations Management, vol. 18, no. 1, pp. 75-93, 1999, doi: 10.1016/S0272-6963(99)00010-8.

[8] R. Sawhney, "Interplay between uncertainty and flexibility across the value-chain: Towards a transformation model of manufacturing flexibility," Journal of Operations Management, vol. 24, no. 5, pp. 476-493, 2006, doi: 10.1016/j.jom.2005.11.008.

[9] M. Pérez Pérez, A. B. María Serrano, and M. C. Fernández López, "A review of manufacturing flexibility: Systematising the concept," International Journal of Production Research, vol. 54, no. 10, pp. 3133-3148, 2016, doi: 10.1080/00207543.2016.1138151.

[10] K.-D. Thoben, S. Wiesner, and T. Wuest, "Industrie 4.0' and Smart Manufacturing - A Review of Research Issues and Application Examples," International Journal of Automation Technology, vol. 11, no. 1, pp. 4-16, 2017, doi: 10.20965/ijat.2017.p0004.

[11] E. Oztemel and S. Gursev, "Literature review of Industry 4.0 and related technologies," Journal of Intelligent Manufacturing, vol. 31, no. 1, pp. 127-182, 2020, doi: 10.1007/s10845-018-1433-8.

[12] M. J. Grant and A. Booth, "A typology of reviews: An analysis of 14 review types and associated methodologies," Health Information and Libraries Journal, vol. 26, no. 2, pp. 91-108, 2009, doi: 10.1111/j.1471-1842.2009.00848.x.

[13] D. M. Mertens, "Mixed Methods Evaluation Designs for Systematic Reviews," Mixed Methods Design in Evaluation, pp. 111-132, 2018, doi: 10.4135/9781506330631.n5.

[14] D. Tranfield, D. Denyer, and P. Smart, "Towards a Methodology for Developing Evidence-Informed Management Knowledge by Means of Systematic Review," British Journal of Management, vol. 14, no. 3, pp. 207222, Sep. 2003, doi: 10.1111/1467-8551.00375.

[15] Y. Koren, X. Gu, and W. Guo, "Reconfigurable manufacturing systems: Principles, design, and future trends," Frontiers of Mechanical Engineering, vol. 13, no. 2, pp. 121-136, 2018, doi: 10.1007/s11465-(018-0483-0.

[16] H. A. el Maraghy, "Flexible and reconfigurable manufacturing systems paradigms," Flexible Services and Manufacturing Journal, vol. 17, no. 4 SPECIAL ISSUE, pp. 261-276, 2006, doi: 10.1007/s10696-006-9028-7.

[17] A. Jain, P. K. Jain, F. T. S. Chan, and S. Singh, "A review on manufacturing flexibility," International Journal of Production Research, vol. 51, no. 19, pp. 5946-5970, Oct. 2013, doi: 10.1080/00207543.2013.824627.

[18] M. B. Gerdin, Å. Fast-Berglund, D. Li, and A. Palmquist, "Knowledge Strategies for Organization 4.0 - A Workforce Centric Approach,” in IFIP WG 5.7 International Conference, APMS 2020, Novi Sad, Serbia, vol. 592 IFIP, 2020, pp. 31-36, doi: 10.1007/978-3-030-57997-5_4.

[19] B. Rekiek and A. Delchambre, Assembly line design : The balancing of mixed-model hybrid assembly lines with genetic algorithms. London: Springer-Verlag London Limited, 2006. doi: 10.1007/b138846.

[20] S. J. Hu et al., "Assembly system design and operations for product variety," CIRP Annals - Manufacturing Technology, vol. 60, no. 2, pp. 715-733, 2011, doi: 10.1016/j.cirp.2011.05.004.

[21] G. Michalos, S. Makris, N. Papakostas, D. Mourtzis, and G. Chryssolouris, "Automotive assembly technologies 
review: challenges and outlook for a flexible and adaptive approach,” CIRP Journal of Manufacturing Science and Technology, vol. 2, no. 2, pp. 81-91, 2010, doi: 10.1016/j. cirpj.2009.12.001.

[22] D. Li, A. Fast-Berglund, and D. Paulin, "Current and future Industry 4.0 capabilities for information and knowledge sharing: Case of two Swedish SMEs," International Journal of Advanced Manufacturing Technology, vol. 105, no. 9, pp. 3951-3963, Dec. 2019, doi: 10.1007/S00170-01903942-5/TABLES/4.

[23] P. E. C. Johansson, L. Malmsköld, A. Fast-Berglund, and L. Moestam, "Challenges of handling assembly information in global manufacturing companies," Journal of Manufacturing Technology Management, vol. 31, no. 5, pp. 955-976, Nov. 2020, doi: 10.1108/JMTM-05-20180137/FULL/XML.

[24] P. Isanaka and F. Liou, "The Applications of Additive Manufacturing Technologies in Cyber Enabled Manufacturing Systems," in Proceedings of the Annual International Solid Freeform Fabrication Symposium - An Additive Manufacturing Conference, 2012, pp. 341-353.

[25] S. Kim and B. Jeong, "Mass Customisation Capability Planning with Additive Manufacturing," in IFIP Advances in Information and Communication Technology, vol. 535, 2018, pp. 184-192, doi: 10.1007/978-3-319-99704-9_23.

[26] P. Mell and T. Grance, "The NIST Definition of Cloud Computing," National Institute of Standards and Technology, US Department of Commerce, Gaithersburg, MD, USA, Rep. Special Publication 800-145, Sep. 2011.

[27] X. Xu, "From cloud computing to cloud manufacturing," Robotics and Computer-Integrated Manufacturing, vol. 28, no. 1, pp. 75-86, 2012, doi: 10.1016/j.rcim.2011.07.002.

[28] P. Pace, G. Aloi, R. Gravina, G. Caliciuri, G. Fortino, and A. Liotta, "An Edge-Based Architecture to Support Efficient Applications for Healthcare Industry 4.0,” IEEE Transactions on Industrial Informatics, vol. 15, no. 1, pp. 481-489, 2019, doi: 10.1109/TII.2018.2843169.

[29] B. Bajic, N. Suzic, N. Simeunovic, S. Moraca, and A. Rikalovic, "Real-time Data Analytics Edge Computing Application for Industry 4.0: The Mahalanobis-Taguchi Approach,” Int J Ind Eng Manag, vol. 11, no. 3, pp. 146156, Sep. 2020, doi: 10.24867/IJIEM-2020-3-260.

[30] M. Engelsberger and T. Greiner, "Self-organising service structures for cyber-physical control models with applications in dynamic factory automation a fog/edgebased solution pattern towards service-oriented process automation," in CLOSER 2017 - Proceedings of the 7th International Conference on Cloud Computing and Services Science, no. Closer, 2017, pp. 238-246, doi: 10.5220/0006365502660274.

[31] Q. Tan, Y. Tong, S. Wu, and D. Li, "Modeling, planning, and scheduling of shop-floor assembly process with dynamic cyber-physical interactions: a case study for CPSbased smart industrial robot production," International Journal of Advanced Manufacturing Technology, pp. 3979-3989, 2019, doi: 10.1007/s00170-019-03940-7.

[32] L. Wang and X. V. Wang, Cloud-Based Cyber-Physical Systems in Manufacturing. 2018. doi: 10.1007/978-3-31967693-7.

[33] L. Monostori, "Cyber-physical production systems: Roots, expectations and R\&D challenges," in Procedia CIRP, Jan. 2014, vol. 17, pp. 9-13. doi: 10.1016/j.procir.2014.03.115.

[34] A. Musil, J. Musil, and S. Biffl, "Towards collective intelligence system architectures for supporting multidisciplinary engineering of cyber-physical production systems," in 1st International Workshop on Cyber-Physical Production Systems, CPPS, 2016, pp. 331-368, doi: 10.1109/CPPS.2016.7483918.
[35] L. Monostori et al., "Cyber-physical systems in manufacturing," CIRP Annals - Manufacturing Technology, vol. 65, no. 2, pp. 621-641, 2016, doi: 10.1016/j.cirp.2016.06.005.

[36] R. Badarinath and V. Prabhu, "Advances in internet of things (Io T) in manufacturing," in IFIP Advances in Information and Communication Technology, 2017, vol. 513, pp. 111-118. doi: 10.1007/978-3-319-66923-6_13.

[37] T. Qu, S. P. Lei, Z. Z. Wang, D. X. Nie, X. Chen, and G. Q. Huang, "IoT-based real-time production logistics synchronisation system under smart cloud manufacturing," International Journal of Advanced Manufacturing Technology, vol. 84, no. 1-4, pp. 147-164, 2016, doi: 10.1007/s00170-015-7220-1.

[38] J. Yan, Y. Meng, L. Lu, and L. Li, "Industrial Big Data in an Industry 4.0 Environment: Challenges, Schemes, and Applications for Predictive Maintenance," IEEE Access, vol. 5, pp. 23484-23491, 2017, doi: 10.1109/ ACCESS.2017.2765544.

[39] D. Guo et al., "Towards Assembly 4.0: Graduation intelligent manufacturing system for fixed-position assembly Islands," IFAC-PapersOnLine, vol. 52, no. 13, pp. 1513-1518, 2019, doi: 10.1016/j.ifacol.2019.11.414.

[40] J. Baalsrud Hauge et al., "Digital Twin Testbed and Practical Applications in Production Logistics with RealTime Location Data," Int J Ind Eng Manag, vol. 12, no. 2, pp. 129-140, 2021, doi: 10.24867/IJIEM-2021-2-282.

[41] Y. Cohen, H. Naseraldin, A. Chaudhuri, and F. Pilati, "Assembly systems in Industry 4.0 era: a road map to understand Assembly 4.0," International Journal of Advanced Manufacturing Technology, vol. 0, pp. 40374054, 2019, doi: 10.1007/s00170-019-04203-1.

[42] H. K. Wu, S. W. Y. Lee, H. Y. Chang, and J. C. Liang, "Current status, opportunities and challenges of augmented reality in education," Computers and Education, vol. 62, pp. 41-49, 2013, doi: 10.1016/j.compedu.2012.10.024.

[43] L. Damiani, R. Revetria, and E. Morra, "Safety in industry 4.0: The multi-purpose applications of augmented reality in digital factories," Advances in Science, Technology and Engineering Systems, vol. 5, no. 2, pp. 248-253, 2020, doi: 10.25046/aj050232.

[44] J. Egger and T. Masood, "Augmented reality in support of intelligent manufacturing - A systematic literature review," Computers and Industrial Engineering, vol. 140, no. December 2019, p. 106195, 2020, doi: 10.1016/j. cie.2019.106195.

[45] E. Tzimas, G. C. Vosniakos, and E. Matsas, "Machine tool setup instructions in the smart factory using augmented reality: a system construction perspective," International Journal on Interactive Design and Manufacturing, vol. 13, no. 1, pp. 121-136, 2019, doi: 10.1007/s12008-018-0470-z.

[46] M. Peshkin and J. E. Colgate, "Cobots," Industrial Robot, vol. 26, no. 5, pp. 335-341, 1999, doi: 10.1108/01439919910283722.

[47] F. Ore, L. Hansson, and M. Wiktorsson, "Method for Design of Human-industrial Robot Collaboration Workstations," Procedia Manufacturing, vol. 11, no. June, pp. 4-12, 2017, doi: 10.1016/j.promfg.2017.07.112.

[48] E. Gambao, M. Hernando, and D. Surdilovic, "A new generation of collaborative robots for material handling," Gerontechnology, vol. 11, no. 2, 2012, doi: 10.4017/ gt.2012.11.02.362.776.

[49] O. Salunkhe, O. Stensöta, M. Åkerman, Å. F. Berglund, and P. A. Alveflo, "Assembly 4.0: Wheel hub nut assembly using a cobot,” IFAC-PapersOnLine, vol. 52, no. 13, pp. 1632-1637, 2019, doi: 10.1016/j.ifacol.2019.11.434.

[50] Y. Hu, Y. Wang, K. Hu, and W. Li, "Adaptive obstacle avoidance in path planning of collaborative robots 
for dynamic manufacturing," Journal of Intelligent Manufacturing, 2021, doi: 10.1007/s10845-021-01825-9.

[51] W. Saleem, H. Ijaz, A. Alzahrani, S. Rubaiee, and M. A. Khan, "Assessment of Optimal Production Through Assembly Line-Balancing and Product-Mix Flexibility," International Journal of Engineering \& Technology, vol. 7, no. 4, pp. 32-36, 2018.

[52] P. E. C. Johansson, F. Delin, S. Jansson, L. Moestam, and A. Fast-Berglund, "Global Truck Production - The Importance of Having a Robust Manufacturing Preparation Process," Procedia CIRP, vol. 57, pp. 631-636, Jan. 2016, doi: 10.1016/J.PROCIR.2016.11.109.

[53] O. Salunkhe and Å. F. Berglund, "Increasing operational flexibility using Industry 4.0 enabling technologies in final assembly" in IEEEInternational Conference onEngineering, Technology and Innovation (ICE/ITMC), 2020, pp. 1-5, doi: 10.1109/ICE/ITMC49519.2020.9198630. 